\title{
Study of the electrical activity of limbic brain structures after the destruction of the dorsal amygdalofugal pathway
}

\begin{abstract}
In order to elucidate the causes of irreversible changes in the activity of the hippocampus after the destruction of the dorsal amygdalofugal pathway, limbic brain structures (amygdala, hypothalamus, reticular formation) were recorded before and after electrical stimulation and destruction of the dorsal amygdalofugal pathway. The data obtained indicate that a necessary condition in the mechanisms of regulation of the excitability of neurons in various nuclei of the hypothalamus, amygdala and reticular formation is a functional integrity of dorsal amygdalofuqal pathway.
\end{abstract}

Keywords: limbic brain structures, dorsal amygdalofugal pathway, destruction
Volume 8 Issue I - 2020

\section{Rafiga Mazahir Baghirova}

Department of Medical and Biological Sciences, Azerbaijan State Academy of Physical Education and Sport,Azerbaijan Republic

Correspondence: Rafiga Mazahir Baghirova,Azerbaijan State Academy of Physical Education and Sport, Department of Medical and Biological Sciences, Baku,Azerbaijan Republic, Email rafiga_bagirovl@mail.ru

Received: January 05, 2020 | Published: February II, 2020

\section{Introduction}

Limbic system is an integral functional system and includes the structures differed with their construction. Morphofunctional features of the limbic system in a complete, highly developed form are easier to analyze if the participation of each structure of this system in its formation is revealed. The complexity of the structural organization of the limbic system needs a complex approach to be applied to its investigation. Conflicting views are first of all the results of unsufficient registration of qualitative differences between brain regions united under the name of the limbic system. One of the main structure of the limbic system is hippocampus for which a peculiar (special, own) form of bioelectric activity-theta rhythm is specific. According to the literary data necessary afferent flow(flux) is provided by ascending systems, its transformation into rhythmic activity occurs on a special hippocampal relay that is considered as a pacemaker of hippocampal theta-rhythm. ${ }^{1-4}$ Ascending fibres as a part of the medial- anterior cerebral bundle enter the medial septal nucleus from the subcortical brain structures which play an important role in the mechanisms of formation of rhythmic hippocampal activity. ${ }^{5-9}$

Our previous studies have shown that destruction of the dorsal amygdalofuqal pathway leads to irreversible blockade of hippocampal theta-rhythm as opposed to ventral. ${ }^{10}$ Electro- and chemostimulation of different hypothalamic nuclei, amygdala and reticular formation did not result in the recovery of hippocampal electrogram under damage of dorsal amygdalofuqal pathway. To find out the reasons of irreversible changes in hippocampal EEG after damage of dorsal amugdalofuqal pathway, also the participation of limbic structures (amygdala, hypothalamus, reticular formation) in the formation of rhythmic hippocampal vibrations we registered electrical activity from supraoptic, ventromedial and medial-mammiliar nuclei of hypothalamus, amygdalar baselateral nucleus, reticular formation before and after electrostimulation and destruction of dorsal amygdalofuqal pathway as well.

\section{Methods}

The experiments were carried out on 18 chinchilla rabbits weighing 2,5-3 kg in a chronic experimental conditions. EEG registration was carried out from supraoptic - SO (A-3; L2,2; H15,8), ventromedial -VMH (P1; LO,5; H17) and medial-mamilliar - MM (A-3; L0,5; $\mathrm{H} 18,5)$ nuclei of hypothalamus, basolateral - AB (A-1; L5; H18) nuclei of amygdala and reticular formation-RF (P9; L2,5; H18,2). Electrostimulation and electrolytic destruction of precommissural region of dorsal amygdalofuqal pathway - DAP (A-1; L3,2; H11,5) were carried-out by bipolar electrodes. The electrical stimulation of DAP was carried out with the help of an electric stimulator ESU-1. For irritation, continuous stimulation with rectangular pulses of 60 $300 \mathrm{mkA}$ intensity was used. With an interelectronic resistance of $25 \mathrm{kHz}$, the pulse trace frequency was $5-100 \mathrm{~Hz}$ for $15-30 \mathrm{sec}$. The duration of each pulse is $0,5 \mathrm{msec}$. The electrolytic destruction of DAP was carried out by the action of a direct current of 1,0 mA for 15-25 sec.

\section{Results and discussion}

The registration of the background electrical activity in $\mathrm{SO}, \mathrm{VMH}$, $\mathrm{MM}, \mathrm{AB}$ and RF showed the availability of polymorph, irregular, low amplitude activity combining both the high frequency beta vibrations, individual theta frequencies and slow waves (Figure 1-I).

One-sided electrostimulation of DAP $(50-80 \mathrm{mkA}$, frequency$10 \mathrm{impulse} / \mathrm{sec}$, with duration of stimulus $-0.5 \mathrm{sec}$ ) leads to significant changes in the electrical activity of the brain structures under study. From the first minute of applying electrical stimulus in the electrograms from $\mathrm{SO}, \mathrm{VMH}, \mathrm{MM}, \mathrm{AB}$ and $\mathrm{RF}$ of both ipsi-, and contralateral sides were registered persistent ordered rhythms, occurrence of peak emissions and continuous epileptiform discharges. The registered changes in EEG from subcortical brain structures were observed from the moment of applying electrical stimulus and maintained for 5-10 sec after turning it off. With a further increase 
in the stimulation parameters $(100-200 \mathrm{mkA}$, frequency-20pulses/ $\mathrm{sec}$, duration of stimulus- $0,5 \mathrm{msec}$ ) for $10-15 \mathrm{sec}$ simultaneously synchronized activity in the electrical activity of all the registered structures on the 1-2nd session of stimulation turns into epileptiform from the first minutes of exposure.

Convulsive discharges occur synchronously in all the brain structures under study and maintain for 5-10sec after the elimination of the electrical stimulus (Figure 1-II). Subsequent registration of EEG from SO, VMH, MM, AB and RF showed the presence of polymorphic, deformed, low amplitude activity which was registered for 1-2 minutes, after which there was a complete restoration of EEG activity to the background level.

One-sided electrolytic destruction of DAP from the first minutes caused acute changes in the electrical activity of $\mathrm{SO}, \mathrm{VMH}, \mathrm{MM}, \mathrm{AB}$ and RF. From the first minutes of destruction in all the studied brain structures were registered epileptiform activity which persists for 2-3 hours after coagulation. During the pointed time is sometimes revealed alternation of epidischarges with flattened, low amplitude, slow wave activity. Over time epileptiform activity completely disappears and in the studied brain structures slow wave, flattened, low amplitude activity is registered which well-defined on the ipsilateral destruction side. On the 2-5th days after the destruction of DAP, the suppression of activity covers both the ipsi- and contralateral sides. It should be noted that the effect of suppression of the activity in the subcortical brain structures as a result of damage to the DAP was irreversible: its recovery was not observed even 6 months after the coagulation (Figure 1-III).

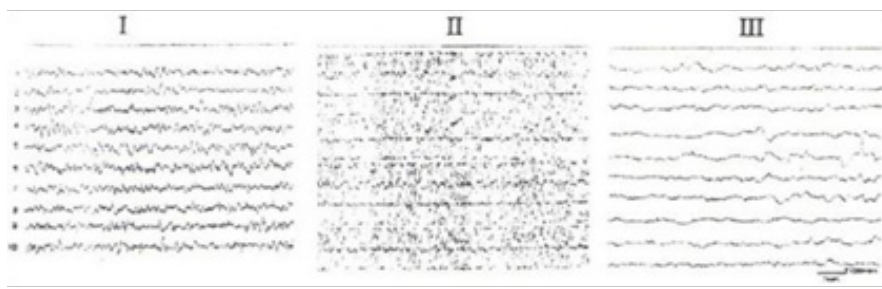

Figure I The effect of stimulation and destruction of DAP on electrica activity of subcortical brain structures.

I-background; II- stimulation moment; III- after the destruction of DAP. I,2 EEG from SO of the hypothalamus, ipsi- and contrlateral side;3,4- EEG from $\mathrm{VMH}$ of the hypothalamus, ipsi-and contrlateral side; 5,6 - EEG from MM of the hypothalamus, ipsi- and contrlateral side; 7,8 - EEG from $A B$ of the amygdala, ipsi- and contrlateral side; 9,I0 - EEG from RF of the middle brain, ipsi- and contrlateral side.

Frequency analysis of the electrogram of the studied brain structures after the destruction of DAP revealed dominance of slow wave alpha and fast beta rhythms, complete disappearance of theta wobble as well. Amplitude analysis showed a sharp decline in the amplitude of EEG activity (Figure 2). It is known that monosynaptic connections of amygdala with hypothalamic formations and other stem structures are brought about by 2 systems of amygdala fibres: dorsal and ventral amygdalefugal pathways. The main part of the afferent fibres of dorsal amygdalofugal pathways passes through central and basolateral amygdalar nuclei and enters front hypothalamus. ${ }^{11}$ Large neurosecretory cell elements of SO are able to synthesize octapeptid vasopressin involved in the secretion of pituitary gland vasopressin, along with corticoliberin, is one of the main stimulants for the release of ACTH from corticotropes. ${ }^{12,13}$ The above mentioned allows to suggest that DAP stimulation appears to result in the activation of hypothalamo-pituitary neurosecretory system, resulting in increased concentration of blood corticosteroid hormones which influence the excitability of the neurons in hypothalamus, amygdala and reticular formation.
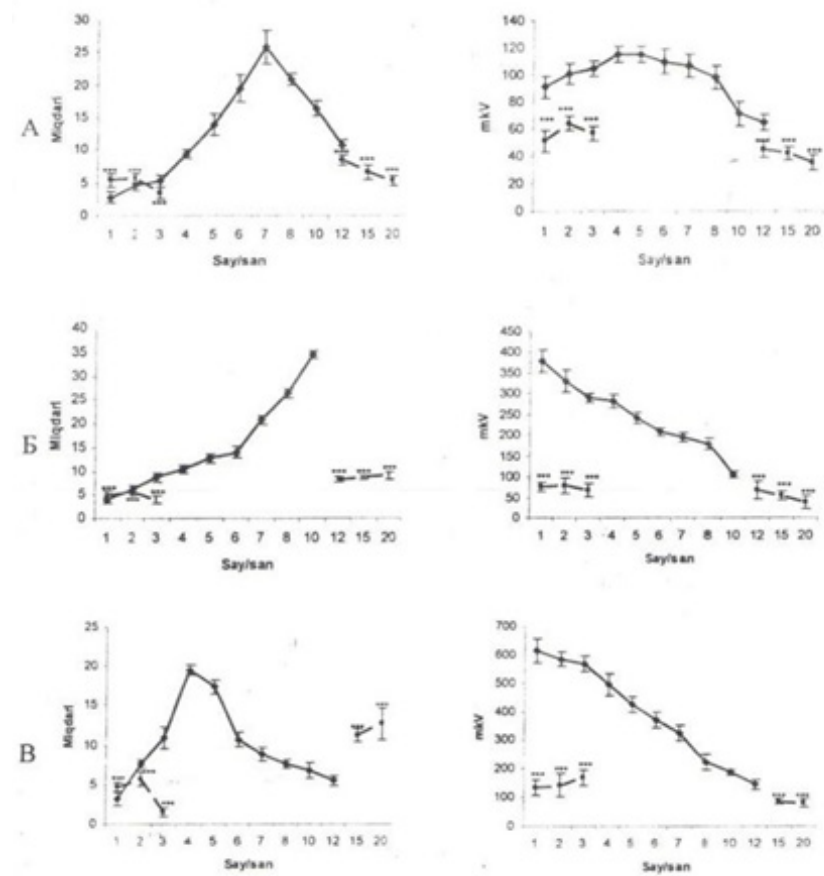

Figure 2 Amplitude -frequency analysis of electrical activity of subcortical brain structures after destruction of DAP well defined on the ipsilateral destruction side.

I, frequency distribution area. The abscissa axis is the average value of class of frequency intervals; The ordinate, the frequency of repeatability; II, Amplitudefrequency response. Solid line- before, dashed- after exposure A, SO of the hypothalamus; $B, A B$ of amygdale; $V$, RF- of the midbrain.

Comparing the data available in the literature on the effect of corticosteroids on the bioelectrical activity of the hypothalamus, almond-shaped complex and reticular formation it was revealed that their action is unidirectional, regardless of the method of hormone administration. So, intramuscular injection of hydrocortisone aids increasing the activity in the posterior, lateral ${ }^{14}$ and anterior medial and arcuat hypothalamic nuclei. ${ }^{15}$ It should be noted that most researchers discovered an activation of excitement process in the hypothalamus under the influence of hydrocortisone which was manifested by amplification of theta- and alpha activities and the occurrence of paroxysmal activity. With administration of ACTH and glucocorticoids the background midbrain electrical activity changes. Intraperitoneal injection of hydrocortisone acetate, also intravenous injection of hydrocortisone or cortisone leads to a noticeable increase in the amplitude of evoked potentials in the midbrain RF and medial hypothalamus. ${ }^{14}$ The direct injection of hydrocortisone into the RF increases noticeably the amplitude of electrical waves in it. The data obtained indicate the possibility of a direct effect of hydrocortisone on the total electrical activity in the midbrain RF which reflects the functional state of the entire brain activating system. Therefore, it is quite natural to assume that one of the mechanisms of activating effect of hydrocortisone is associated with its direct effect on the midbrain RF. ${ }^{12}$

In the amygdala changes in the electrical activity are also registered with the application of hydrocortisone, cortisone-acetate 
and desoxycorticosterone into basolateral and cortical nuclei of the almond-shaped complex. Mikroinjection of corticosterone into amygdala leads to increase in the amplitude of electrical activity of its main rhythm. The application of corticosterone into the amygdala leads to a decrease in the activity of this structure. ${ }^{15}$ Comparing the data available data in the literature with ours one can suggest that the stimulation of DAP both with low and increased parameters of the stimulating current leads to an increase in the concentrations of corticosteroid hormones in the blood which increase the excitability of neurons from different nuclei of the hypothalamus, amygdala and reticular formation and registration in their activity synchronized theta-waves at low stimulating currents and epileptiform activity with increasing parameters of irritation. Our suggestion is broadly consistent with the data available in the literature which testifies an increase in ACTH in the blood of the jugular vein and 17-oxicorticosteroids in the blood of the renal vein during stimulation of the terminal strip. ${ }^{16,17}$

Damage of DAP results in irreversible changes in the electrical activity of different nuclei of hypothalamus, amygdala and reticular formation which manifested in the registration of irregular, high frequency, low amplitude activity. Considering regular effect of the amygdale on the functional state of hypothalamic nuclei, ${ }^{18-20}$ as well as availability direct relations with neurosecretary cells of the hypothalamus one can suggest that hypothalamus gets out of amygdalar control after the destruction of DAP, activity of the hypothalamic-pituitary-adrenal system is disturbed that leads to increase in the amount of ACTH and steroid hormones which over the course of 2-3 hours lead to the registration of epileptiform activity in different nuclei of hypothalamus, amygdale and reticular formation, with followed by cell death of these structures which reflects in the blockade of their electrical activity. The validity of our research is consistent with the available data indicating that under conditions of deafferentation, the functional activity of the hypothalamic-pituitaryadrenal system is disturbed, i.e. it is reduced by about half. ${ }^{11}$

Thus, one can be concluded that a necessary condition in the mechanisms of regulation of the excitability of neurons in various nuclei of the hypothalamus, amygdala and reticular formation is a functional integrity of dorsal amygdalofuqal pathway.

\section{Acknowledgments}

None.

\section{Conflicts of interest}

Author declares that there is no conflict of interest.

\section{References}

1. Kitchigina VF. Regulation mechanisms and functional significance of theta oscillations in the septo-hippocampal system of the brain. Abstract of dissertation for the degree of Doctor of Biological Sciences: Moscow, Pushchino; 2006.

2. Kichigina VF, Kutyreva EV. Modulation of theta activity in septohippocampal system alpha-2-adrenoceptoragonist clonidine. Zh Vyssh Nerv Deiat Im I P Pavlova. 2002;52(2):195-204.
3. Kitchigina V, Popova I, Sinelnikova V, et al. Disturbances of septohippocampal theta oscillations in the epileptic brain: Reasons and consequences. Experimental Neurology. 2013;247:314-327.

4. Mysin IE, Kitchigina VF, Kazanovich Y. Modeling synchronous theta activity in the medial septum: key role of local communications between different cell populations. J Comput Neurosci. 2015;39(1):1-16.

5. Berridge CW, Isaak SO, Espana RA. Additive wake-promoting actions of medial basal forebrain noradrenergic alphal-and beta-receptor stimulation. Behav Neurosci. 2003;117(2):350-359.

6. Kichigina VF, Kudina TA. Sensory reaction of hippocampal neurons of the rabbit under functional you turn off the structures that control theta rhythm. Zh Vyssh Nerv Deiat Im I P Pavlova. 2001;51(2):228-235.

7. Kichigina VF, Kutyreva EV. Modulation of theta activity in septohippocampal system alpha-2-adrenoceptoragonist clonidine. Zh Vyssh Nerv Deiat Im I P Pavlova. 2002;52(2):195-204.

8. Smythe JW, Cristie BR, Colom LV, et al. Hippocompal theta field activity and thetaon/theta-off all discharges are controlled by an ascending hypothalamo-septal pathway. J Neuroscience. 1991;11(7):2241-2248.

9. Steriade M. Arousal: revisiting the reticular activating system. Science. 1996;272(5259):225-226.

10. Gasanov GG, Kasimov AÉ, Bagirova RM. Contributions of the amygdala and hypothalamus to the formation of hippocampal theta activity. Nauchnye Doki Vyss Shkoly Biol Nauki. 1989;3:51-56.

11. Chepurnov SA, Chepurnova NE. The amygdala complex of the brain. Moscow Publishing House: Univ; 1981. 256 p.

12. Sapronov NS. Hormones of the hypothalamic-pituitary-ovarian system and the brain. St. Petersburg: Formizdat; 2009. 591 p.

13. Linton EA, Gilles G, Lowry PJ. Ovine corticotropin- releasing factor and vasopressin: antibody-cuenching studies on hypothalamic extract of normal and Brattleboro rats. Endocrinology. 1983;113(5):1878-1883.

14. Feldeman S, Todt JC Porter RW. Effect of adrenocitrial hormones on evoked potentials in the brain steam. Neurology. 1961;11:109-115.

15. Malyshenko NM. The effect of hydrocortisone on the hypothalamichippocampal relationship. Bulletin of Experimental Biology and Medicine. 1973;76(1):798-803.

16. Okinaka S, Ibayaschi H, Notohaschi K, et al. Regulation of the pituitaryadrenocortical function through limbic system and reticular formation. Acta endocrinol. 1965;35(51):43-44.

17. Salkman N, Peck L, Egdahl RH. Effect of acute and prolonget electrical stimulation of the amygdala of the dog upon peripheral plasma concentration of corticosteroids. Neuroendocrinology. 1970;6(5):361-367.

18. Olmos JS, de Ingram VR. The projection field of the stria terminalis in the rat brain: An experimental study. J Comp Neurol. 1972;146(3):303-334.

19. Steriade M. Arousal: revisiting the reticular activating system. Science. 1996;272(5259):225-226.

20. Vertes RP, Kocsis B. Brainstem-diencephalo-septo-hippocampal system controlling the theta rhythm of the hippocampus. $J$ Neuroscience. 1997;81(4):893-926. 